\title{
A PERCEPÇÃO DE MULHERES IDOSAS SOBRE O IMPACTO DO TREINAMENTO FISICO NAS SUAS ATIVIDADES DE VIDA DIÁRIA?
}

\author{
Célia Pereira Caldas² \\ Celia Cohen Barros ${ }^{3}$
}

resumo

É fato que o exercício físico em geral traz efeitos benéficos ao desempenho físico de pessoas idosas, o que aumenta o empenho dos estudiosos na busca de mais informações sobre sua utilização e seus benefícios nos variados campos da saúde. O objetivo do presente estudo é avaliar a percepção de mulheres idosas quanto às modificações provocadas pelos exercícios físicos em suas atividades de vida diária. Este estudo é o componente qualitativo de um estudo de intervenção, com uma população de idosas voluntárias, realizado nas seguintes etapas: uma avaliação prévia, uma intervenção, seguindo-se uma pesquisa qualitativa de análise de conteúdo sobre a percepção das idosas. Foram estudadas 90 mulheres idosas

\footnotetext{
1 O presente estudo foi financiado pela fundação Capes (Coordenação de Aperfeiçoamento de Pessoal de Nível Superior).

2 Graduada em Enfermagem. Doutora em Enfermagem. Professora Associada da Universidade do Estado do Rio de Janeiro (UERJ), vinculada ao Departamento de Saúde Pública. E-mail: ccaldas@uerj.br. 3 Graduada em Educação Física. Doutora em Ciências Médicas. Profissional liberal atuando em treinamento físico para pessoas idosas. E-mail: celiacohen@uol.com.br.
} 
fisicamente independentes, não praticantes de exercícios físicos. As voluntárias foram submetidas a 24 sessões de treinamento. Na pesquisa qualitativa, as voluntárias demonstraram em suas falas a importância do treinamento para obter melhoras na dimensão física, psíquica, social e cognitiva. Conclui-se que as idosas percebem as modificações produzidas pelo treinamento físico.

palavras-chave

Idoso. Envelhecimento. Exercício Físico. Pesquisa Qualitativa.

\section{Introdução}

A mudança no padrão demográfico do Brasil, em relação à idade da população, é um fato. A transformação, iniciada gradativamente por volta da década de 1960, fez com que a população com mais de 60 anos de idade aumentasse de forma expressiva. No censo demográfico populacional realizado pelo Instituto Brasileiro de Geografia e Estatística (IBGE, 2010), em 2000, eles representavam 13.915 .357 pessoas, correspondendo a $8,41 \%$ do total da população brasileira. O censo de 2010 mostrou que os idosos são em número de 20.590.599, correspondendo a 11,0\% do total da população brasileira. De acordo com a projeção populacional brasileira realizada pelo mesmo Instituto, a expectativa projetada é de um aumento aproximado de 64.050 .979 ou $29,72 \%$ até o ano de 2050 (IBGE, 2010).

Essa transição demográfica acentuada na população brasileira ocasiona dificuldades para o governo se adaptar a esse novo panorama que, consequentemente, gera impacto social, cultural, político e econômico muito grande, uma vez que aumenta o número de "inativos" de forma desproporcional ao número da população economicamente ativa. O governo terá que promover programas para aumentar os recursos econômicos, prover assistência médica, previdenciária e de socialização digna aos cidadãos que envelhecem para que eles não fiquem à margem da sociedade e sejam vistos como pessoas que podem ser úteis e capazes de produzir (VERAS, 1994; KALACHE, 1996).

Considera-se o envelhecimento um processo dinâmico, contínuo e fisiológico, ocorrendo modificações morfológicas, funcionais, psicológicas e bioquímicas, acompanhadas por diminuição da capacidade funcional que, em longo 
prazo, determina redução importante do potencial de adaptação do indivíduo ao ambiente e ao cotidiano, sendo maior a incidência de processos patológicos crônicos que acabam por resultar em morte (LIBERMAN, 2011; BARROS; CALDAS; BATISTA, 2013). Por esse motivo, na busca de melhor qualidade de vida, é importante proporcionar e incentivar a população idosa a se manter saudável, produtiva e independente pelo maior número de anos possível.

Essa tendência global tem levado a ciência, os pesquisadores e a população em geral a procurar cada vez mais soluções para tentar minimizar ou, se possível, evitar os efeitos negativos do avanço da idade cronológica no organismo. Cada vez mais se pesquisam formas de deter ou retardar o processo de envelhecimento, de forma a garantir a manutenção da capacidade funcional e a autonomia nas últimas décadas de vida.

A literatura conta com muitos estudos sobre a relação entre nível de atividade física e prevalência de fatores de risco para doenças crônico-degenerativas, com resultados que demonstram correlação inversa entre elas (BENEKA et al., 2005; PALATINI et al., 2009). Nesse sentido, acumulam-se pesquisas que procuram estabelecer uma relação entre a prática regular de atividades físicas e aspectos como longevidade, prevenção, controle e tratamento das principais doenças crônicas associadas ao processo de envelhecimento, além dos possíveis efeitos sobre os aspectos psicológicos, sociais e cognitivos dos indivíduos idosos (HAMER; STAMATAKIS, 2009; AMERICAN COLLEGE OF SPORTS MEDICINE, 2009). Tendo em vista que grande parte das evidências dá apoio à ideia de que um estilo de vida ativo contribui para a prevenção e minimização dos efeitos negativos do envelhecimento, a prática de exercícios físicos de forma permanente vem sendo valorizada no âmbito de programas que visam a promover a saúde do idoso para que ele possa, efetivamente, viver a terceira idade (AMERICAN COLLEGE OF SPORTS MEDICINE, 2009).

A melhoria e a manutenção da capacidade física com a prática de exercícios físicos tornam-se necessárias porque contribuem com a autonomia funcional do idoso. Segundo muitos estudiosos, a regularidade da prática de exercícios físicos melhora o condicionamento físico e sua socialização (GOBBI; VILLAR; ZAGO, 2005; SILVA et al., 2006; AMERICAN COLLEGE OF SPORTS MEDICINE, 2009; METSIOS et al., 2009).

O objetivo básico da prática regular de exercícios físicos é preservar a autonomia e a independência, refletindo positivamente nos níveis da saúde como um todo. É de fundamental importância o incentivo à conscientização do idoso para a percepção dos benefícios, em todos os aspectos, trazidos pelo treinamento sistematizado, uma vez que esse fator poderá ser determinante na sua aderência ao programa de exercícios. 
Dessa forma, é necessário que programas adequados de condicionamento físico sejam elaborados e realizados, respeitando as características e necessidades individuais dos idosos, que devem ser considerados como seres humanos envolvidos no processo do estudo, e não apenas serem vistos por suas limitações ou seu estado especial. Nesse sentido, o presente estudo procurou contribuir para a produção de conhecimento científico, verificando os benefícios do treinamento físico para a população estudada, levando em consideração não só os resultados quantitativos, mas também de que forma essas modificações foram percebidas pelas voluntárias.

\section{Métodos}

O presente estudo é o componente qualitativo de um estudo misto, que foi iniciado por um estudo de intervenção, com uma população de idosas voluntárias. A pesquisa foi realizada no período de 2009 a 2014, no Instituto de Educação Física e Desportos (IEFD), da Universidade do Estado do Rio de Janeiro (UERJ). Este estudo foi submetido à apreciação do Comitê de Ética em Pesquisa do Hospital Universitário Pedro Ernesto da Universidade do Estado do Rio de Janeiro (HUPE/UERJ), de acordo com a Resolução CNS 466/12 e aprovado, sob o no 2489 - CEP/HUPE - CAAE: 0072.0.228.000-09.

Foram estudadas 90 mulheres voluntárias, idosas, oriundas do Centro de Convivência da Universidade Aberta da Terceira Idade da Universidade do Estado do Rio de Janeiro (UNATI/UERJ), que atenderam aos seguintes critérios de inclusão: idade $\geq 60$ anos; fisicamente independentes; não praticantes de exercícios físicos e aptas a realizar todos os exercícios e testes propostos. A decisão por estudar apenas mulheres deve-se ao fato do quantitativo de voluntários homens frequentadores do centro de convivência não ter sido suficiente para formar um grupo estatisticamente significativo para o estudo.

Foram considerados critérios de exclusão: portadoras de doenças articulares ou musculares, deficiências físicas ou mentais, doenças cardíacas. E, durante o período de treinamento, apresentar número superior a três faltas consecutivas ou cinco alternadas em todo o período de treinamento, assim como faltar às avaliações.

A intervenção incluiu 24 sessões de treinamento de intensidade moderada, executados três vezes por semana em dias alternados. As sessões, com duração aproximada de 60 minutos, foram compostas por exercícios de força, flexibilidade e resistência aeróbia. 
Para melhor compreender o significado do resultado da intervenção na população estudada, foi realizado um estudo qualitativo, utilizando o método de análise de conteúdo segundo Bardin (2007). A entrevista foi conduzida por outro pesquisador, diferente do pesquisador que realizou o treinamento, e desconhecido das voluntárias.

As participantes submetidas a treinamento responderam, inicialmente, a uma pergunta: “Você sentiu alguma diferença no seu dia a dia após o período de treinamento?". A pergunta foi respondida livremente sem limitação de tempo. Em alguns momentos houve necessidade de interferência do entrevistador para solicitar esclarecimento sobre algum ponto ou aprofundamento de algum detalhe que chamasse a atenção. As entrevistas foram transcritas, respeitando-se as construções das frases, os erros gramaticais, as repetições, as pausas e os titubeios ocorridos durante a fala.

A partir de uma leitura flutuante de todos os depoimentos, cada resposta foi trabalhada individualmente, quando foram feitas leituras e releituras dos discursos para uma familiarização com o texto e para um entendimento sobre o que as voluntárias buscavam transmitir, a fim de se identificar as características comuns, revelando-se as categorias temáticas nos discursos. Posteriormente, foram assinaladas frases e trechos significativos para a compreensão do fenômeno pesquisado.

\section{Resultados}

Após a leitura e análise do conteúdo das respostas, observou-se que todas relataram ter havido alguma mudança para melhor em uma ou mais categorias emergentes. Foram encontradas três categorias centrais, cada uma com suas subcategorias.

Na Figura 1 são apresentados os resultados da análise descritiva de acordo com as categorias e subcategorias identificadas na entrevista. 
Figura 1 - Fluxograma das categorias e subcategorias emergentes.

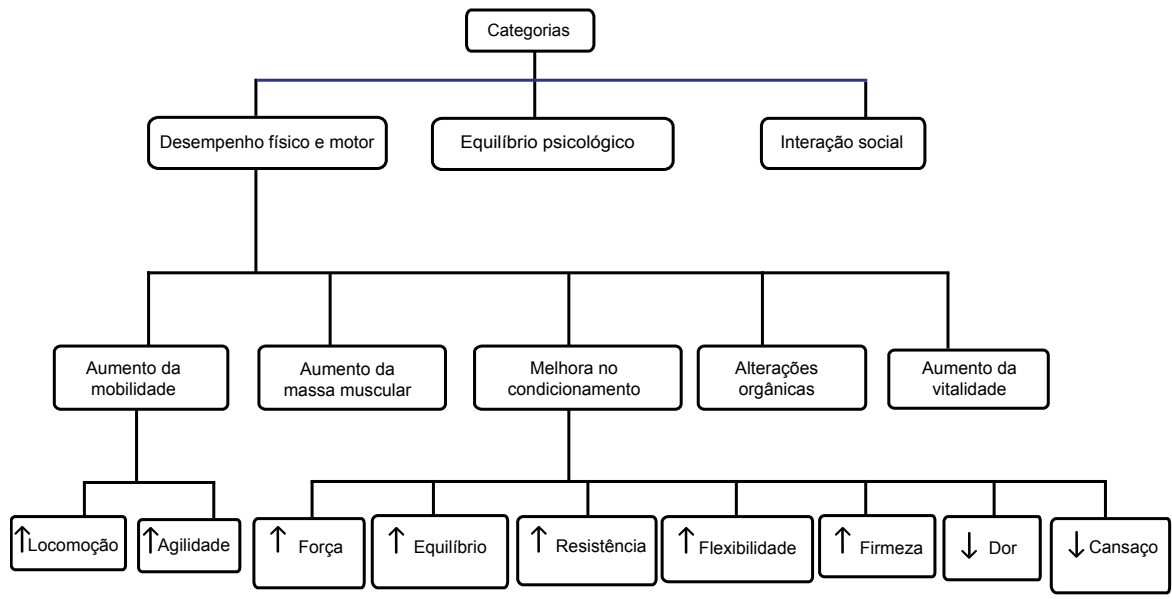

Fonte: Elaborada pelas autoras.

As mudanças relatadas se deram principalmente em relação ao desempenho físico e motor, razão pela qual a categoria 1- desempenho físico e motor apareceu com maior destaque e desdobrou-se em cinco subcategorias: aumento da mobilidade; aumento da massa muscular; melhora no condicionamento; alterações orgânicas e aumento da vitalidade. Em relação ao aumento da mobilidade, observou-se que as mulheres destacaram a locomoção e a agilidade como maiores ganhos: $74 \%$ relataram apresentar melhoras na sua locomoção e $12 \%$ relataram melhoras na sua agilidade. Em relação à massa muscular, $23 \%$ perceberam aumento. $\mathrm{O}$ reconhecimento destas mudanças pode ser verificado nos seguintes depoimentos:

"Melhorei muito depois que comecei a fazer a musculação, antes não conseguia andar muito, tinha que parar porque sentia dor nas pernas, e depois que comecei a musculação conseguia andar muito mais tempo sem parar..."

"Senti melhora nos movimentos, eu estava com dificuldades nos movimentos, não consegui levantar, agachar e com esses exercícios eu senti que melhorei bastante! Melhorei muito minha mobilidade, agachar, amarrar meu sapato, que eu tinha dificuldade, principalmente o tênis..."

"Me senti mais ágil! Eu percebi quando eu me levantava do sofá..."

"Notei o enrijecimento da musculatura, eu tenho visto o efeito..." 
"Senti muita diferença, conheci novas pessoas, minhas pernas estão duras e ficaram bem fortes em pouquinho tempo! No dia a dia faço tudo e fiquei mais ativa, com mais força..."

Quanto à melhora no condicionamento, houve relato de aumento na força (24\%); equilíbrio (6\%); resistência (15\%); flexibilidade (5\%); e firmeza (14\%). Houve relato de redução nas dores em 39\% das mulheres e redução no cansaço em $20 \%$. Os depoimentos que se seguem atestam a melhora percebida pelas depoentes:

"No dia a dia senti mais disposta e maior facilidade de carregar sacolas, ia direto até em casa sem precisar parar pra descansar..."

"Senti muita melhora nas pernas, nos braços, mais força, mais firmeza, antes eu tropeçava, agora estou mais firme!..."

"Eu senti tudo, animação, estou andando melhor, eu sentia que ia cair e agora estou mais firme, firmeza no corpo todo, antes eu caía muito!..."

"Mas na rua ando com mais firmeza! Eu ando muito, vou na UNATi todo dia... Pego trem, eu moro em Bento Ribeiro..."

"No corpo melhorei das dores, nas atividades diárias tenho mais vontade de fazer as coisas..."

"Eu me sinto mais disposta! Faço minhas coisas com facilidade, sem cansaço! Antes eu ficava com preguiça! Ficava dentro de casa naquela moleza..."

Quanto às alterações orgânicas, 36\% perceberam alterações positivas orgânicas hemodinâmicas ou metabólicas e $55 \%$ perceberam aumento da vitalidade, como se pode verificar nos seguintes depoimentos:

"Eu não poderia imaginar que aquela meia horinha, carregando aquele peso! Que minha pressão fosse normalizar daquele jeito!..."

"E até emagreci um pouco, perdi dois quilos sem fazer dieta! Eu gostei!..."

"E minha qualidade de sono melhorou muito!..."

"Passei a dormir melhor, eu também senti que tinha uma bola de gordura no braço, e agora está desaparecendo com o exercício, e em casa me sinto bem mais disposta..."

Na categoria 2- equilíbrio psicológico, $72 \%$ perceberam melhoras em algum aspecto psicológico. Os depoimentos abaixo ilustram o ganho de equilíbrio psicológico percebido pelas mulheres que participaram do programa de treinamento. 
"Me sinto mais ativa, acordo cedo, lá tem o medicamento para minha depressão, minha síndrome do pânico. Lá eu tenho o medicamento que não encontro em nenhuma farmácia! Eu saio de lá feliz, é um medicamento que não encontro em nenhuma farmácia!..."

"Minha autoestima melhorou muito!..."

"E a parte mental também melhorou muito! Porque ali também é uma distração, e você se sentindo melhor a sua mente também parece que fica melhor!..."

Na categoria 3- interação social, $25 \%$ perceberam melhoras na sua interação social, como pode ser verificado nos depoimentos das participantes:

"O que gostei mais foi da farra! Das "meninas". Esse treinamento me trouxe a maior alegria! Era a maior alegria na hora de me arrumar pra vir pra cá! Ficava doida pra vir pra cá!..."

"A interação com as pessoas foi muito boa, é um grupo muito interessante, é até uma terapia, apesar de o objetivo não ser esse..."

"A turma também e as amigas, o convívio foi maravilhoso com todas, e eu tenho o prazer de acordar e vir cedo e feliz!..."

Em todas as categorias e subcategorias é possível identificar nos depoimentos uma certa surpresa em relação aos resultados positivos alcançados com o treinamento físico. As participantes do estudo reconhecem e apontam claramente que os benefícios percebidos foram além até da sua expectativa inicial ao ingressar no programa.

\section{Discussão}

Quando analisadas as categorias separadamente para o desempenho físico/motor, elas relataram terem percebido grande melhora na mobilidade constatada pela facilidade de execução nas suas tarefas diárias e também na capacidade de locomoção, observada pela melhora no caminhar, subir escadas, subir/descer numa condução ou se locomover para lugares diferentes. Fato bastante importante, pois essa melhora tem interferência direta na vida do idoso tornando-o mais independente e seguro para um convívio mais ativo na sociedade (AMERICAN COLLEGE OF SPORTS MEDICINE, 2009).

A melhora na agilidade também foi percebida pelas idosas de todos os grupos que relataram se sentir mais ágeis no seu dia a dia. A percepção das idosas para o aumento da massa muscular revelou íntima relação com o aumento da força (AMERICAN COLLEGE OF SPORTS MEDICINE, 2009; BARROS; CALDAS, 2016). 
Um fato bastante importante que se destacou foi a percepção das idosas para o aumento da massa muscular, relacionando-a à estética corporal. Ou seja, elas tiveram a capacidade de perceber modificações na imagem corporal, melhorando o conceito que faziam de si próprias, fato que apresentou repercussão direta sobre a autoestima de cada uma (OKUMA, 2004). Já o aumento da força, as idosas relataram ter percebido quando realizavam determinadas tarefas do dia a dia como, por exemplo, carregar sacolas do mercado ou segurar o neto no colo (AMERICAN COLLEGE OF SPORTS MEDICINE, 2009; BARROS; CALDAS; BATISTA, 2013; BARROS; CALDAS, 2016).

A melhora na flexibilidade, na firmeza e no equilíbrio também foi relatada pelas idosas, principalmente na realização das tarefas diárias e de autocuidado, mas também em situações de risco como as de quedas. As idosas relataram se sentir seguras e mais confiantes para sair à rua.

$\mathrm{O}$ aumento da resistência e a redução do cansaço também apareceram nos discursos como uma capacidade de resistir a esforços mais prolongados tais como tarefas domésticas e de cuidadores, e também no lazer (BARROS; CALDAS, 2016). A expressão redução da dor foi bastante mencionada pelas idosas e apareceu como importante fator na valorização do exercício físico, influenciando em outros fatores como a resistência a esforços contínuos e repetidos (AMERICAN COLLEGE OF SPORTS MEDICINE, 2009).

As idosas relataram perceber modificações orgânicas tais como regularização no sono, no aparelho digestivo e circulatório, notaram emagrecimento, relataram que normalizaram a pressão arterial, melhora nas taxas do colesterol e redução da medicação. É importante ressaltar que no presente estudo nenhuma dessas variáveis foi avaliada, porém essas modificações foram por elas percebidas (SILVA et al., 2006; AMERICAN COLLEGE OF SPORTS MEDICINE, 2009).

O aumento da vitalidade que apareceu com alta frequência no discurso das idosas foi interpretado como um conjunto de benefícios associados que, muitas vezes, as voluntárias não conseguiam expressar de forma clara, relatando que havia aumentado a disposição sem explicar qual o significado tanto da disposição quanto da vitalidade (AMERICAN COLLEGE OF SPORTS MEDICINE, 2009). O cognitivo aparece de forma muito sutil em dois relatos nos quais as idosas explanaram sobre a melhora da sua memória e sobre o aprendizado de coisas novas.

Os aspectos existenciais e o equilíbrio psicológico emergiram com muita ênfase para os três grupos. As idosas relataram o quanto o exercício físico contribuiu para a redução das angústias, da depressão e das tristezas que foram 
transformadas em bem-estar, prazer, autoestima, alegria de viver, confiança e autovalorização (OKUMA, 2004).

A interação social também foi um aspecto que emergiu em destaque nos discursos. A possibilidade de desfrutar do convívio com semelhantes mostrou que foi uma experiência significativa naquele momento de vida. Essa possibilidade permitiu ao idoso ter uma rede de ligações pessoais, na qual poderia trocar afeto, acolhimento, conhecimentos, experiências de vida, sentir-se importante, valorizado e amado (OKUMA, 2004).

De forma mais específica, na interação social apareceu com grande frequência o relacionamento com o profissional que conduzia o estudo; os relatos mostraram o tamanho da importância dessa interação para o sucesso dos resultados. Nas falas, o modo como o professor se relacionava com as idosas demonstrou que esse relacionamento foi marcante na experiência vivida durante o estudo. O papel do professor foi claramente explicitado como de grande importância na motivação e na construção de laços de afeto e acolhimento. $\mathrm{O}$ atendimento individualizado e a adequação das tarefas às possibilidades de cada um levaram a um sentimento de acolhimento e motivação e, dessa forma, os idosos se sentiam competentes na realização das tarefas propostas, sentimentos esses fundamentais para despertar o interesse pelo que faziam (OKUMA, 2004). É importante ressaltar que as idosas perceberam que não podiam mais deixar de praticar a atividade física, pois isso significaria retornar às condições de saúde mais precárias, ao desânimo, à ausência de interesse para se ocupar, ao cansaço, à lentidão e à dificuldade de realizar suas tarefas.

\section{Conclusão}

As idosas foram capazes de reconhecer a importância do treinamento realizado para obter melhoras na dimensão física, psíquica, social e cognitiva. Considerando-se o relato das idosas sobre os benefícios trazidos pelo treinamento físico, verificou-se que o universo do estudo é bem mais amplo do que possa estabelecer uma simples relação numérica. O fato de conhecer os resultados pela perspectiva de quem sofre a ação, estreita o relacionamento entre as partes (pesquisador e pesquisado) o que propicia uma maior compreensão dos fenômenos ocorridos e a facilitação da obtenção de melhores resultados. Tal fato possibilita refletir o quanto é importante levar em consideração a experiência do idoso no estudo da atividade física. 


\section{ELDERLY WOMEN'S PERCEPTION \\ OF PHYSICAL TRAINING IMPACT \\ ON DAILY LIVING ACTIVITIES}

abstract

It is a fact that exercise generally brings benefits to the physical performance of older people, which increases the commitment of scholars in search of more information on its use and its benefits in various fields of health. The aim of this study is to evaluate the perception of elderly women as to any changes caused by exercise in their daily activities. This study is the qualitative component of an intervention study with a population of elderly volunteers performed the following steps: a preliminary assessment, intervention, followed by a qualitative research content analysis on the perception of the elderly. 90 physically independent older women, not practicing physical exercise were studied. The volunteers were subjected to 24 training sessions. In qualitative research, the volunteers demonstrated in their speeches the importance of training for improvements in physical, mental, social and cognitive. We conclude that elderly perceive the changes produced by physical training.

keywords

Elderly People. Aging. Physical Exercise. Qualitative Research.

referências

AMERICAN COLLEGE OF SPORTS MEDICINE. American College of Sports Medicine position stand. Exercise and physical activity for older adults. Medicine \& Science in Sports \& Exercise, Indianapolis, v. 41, n. 7, p. 1510-1530, July 2009.

BARDIN, Laurence. Análise de conteúdo. 3. ed. Lisboa: Edições 70, 2007.

BARROS, Celia Cohen; CALDAS, Célia Pereira. Strength, power and resistance training in older women. Health, Irvine, v. 8, n. 6, p. 575-582, Jan. 2016.

BARROS, Celia Cohen; CALDAS, Célia Pereira; BATISTA, Luiz Alberto. Influence of muscle power training on the ability to implement motor tasks for older women. Revista Brasileira de Geriatria e Gerontologia, Rio de Janeiro, v. 16, n. 3, p. 603-613, 2013.

BENEKA, Anastasia et al. Resistance training effects on muscular strength of elderly are related to intensity and gender. Journal of Science and Medicine in Sport, Melbourne, v. 8, n. 3, p. 274-283, 2005

GOBBI, Sebastião; VILLAR, Rodrigo; ZAGO, Anderson Saranz. Bases teórico-práticas do condicionamento físico. Rio de Janeiro: Guanabara Koogan, 2005.

HAMER, Mark; STAMATAKIS, Emmanuel. Physical activity and mortality in men and women with diagnosed cardiovascular disease. European Journal of Cardiovascular Prevention \& Rehabilitation, London, v. 16, n. 2, p. 156-160, 2009. 
INSTITUTO BRASILEIRO DE GEOGRAFIA E ESTATÍSTICA (IBGE). Censo demográfico 2010. Rio de Janeiro: IBGE, 2010. Disponível em: http://censo2010.ibge.gov.br. Acesso em: 24 fev. 2014

KALACHE, Alexandre. Envelhecimento no contexto internacional: a perspectiva da Organização Mundial da Saúde. In: SEMINÁRIO INTERNACIONAL SOBRE ENVELHECIMENTO POPULACIONAL: UMA AGENDA PARA O FINAL DO SÉCULO, 1., 1996, Brasília, DF. Anais [...]. Brasília, DF: Secretaria da Assistência Social, Ministério da Previdência e Assistência Social, 1996. p. 13-15.

LIBERMAN, Sami. Envelhecimento do sistema endócrino. In: FREITAS, Elizabete Viana et al. (org.). Tratado de geriatria e gerontologia. Rio de Janeiro: Guanabara Koogan, 2011. p. 788-795.

METSIOS, George et al. Association of physical inactivity with increased cardiovascular risk in patients with rheumatoid arthritis. European Journal of Cardiovascular Prevention \& Rehabilitation, London, v. 16, n. 2, p. 188-194, 2009.

OKUMA, Silene Sumire. O idoso e a atividade física: fundamentos e pesquisa. 3. ed. Campinas: Papirus, 2004.

PALATINI, Paolo et al. Regular physical activity prevents development of left ventricular hypertrophy in hypertension. European Heart Journal, London, v. 30, n. 2, p. 225$-232,2009$

SILVA, E. et al. Efeito agudo e crônico do treinamento físico aeróbio sobre a resposta da pressão arterial sistêmica de indivíduos hipertensos. Revista da Sociedade de Cardiologia do Estado de São Paulo, São Paulo, n. 16, p. 9-20, 2006.

VERAS, Renato Peixoto. País jovem com cabelos brancos: a saúde do idoso no Brasil. Rio de Janeiro: Relume-Dumará, 1994. 